\title{
Akshay Kumar Chakravarthy and Shakunthala Sridhara (eds): Economic and ecological significance of Arthropods in diversified ecosystems. Sustaining regulatory mechanisms
}

\author{
Springer, Singapore 2016, xxii $422 \mathrm{pp}, 51 \mathrm{~b} / \mathrm{w}$ illustrations, 122 illustrations in colour, \\ £119.00 (hardcover), ISBN 978-981-10-1523-6
}

\section{Tom Brereton ${ }^{1}$}

Published online: 25 July 2017

(C) Springer International Publishing AG 2017

This book is a composite of papers from an International Conference on Insect Science in 2013. It chiefly covers the status, function, importance and control of insects and other Arthropods in agricultural systems. The conference was held in India, which is reflected in the content of the book with 53 of the 62 contributions from Indian researchers and much of the remainder from south-east Asia and Australasia. There are no European or American contributions, though there is one from Iran.

The book starts with introductory chapters on Arthropod taxonomy and diversity and their vital role in maintaining and regulating soil fertility. The next 3 chapters describe the diversity of insect communities in various regions and agricultural systems of the Middle East and Asia. Chapters 6 and 7 are very interesting summaries of the utilitarian use of insects as food and in medicine, including within indigenous communities.

The main body of the book (Chaps. 8-18) is a series of systematic reviews of Arthropods in various traditional and modern agricultural and horticultural crop systems-their diversity and positive role in ecosystem function, and negative role as pests and vectors of disease. Control measures are described. Most major tropical and sub-tropical crop types of south-east Asia are covered, including tea, coffee, rice, cotton, mango, vegetables and a wealth of information is presented.

A recurrent theme is the negative impact of intensive crop monocultures on Arthropod diversity and conservation and, in some cases, in terms of indirectly facilitating population booms in pest species. The value of traditional systems

Tom Brereton

tbrereton@butterfly-conservation.org

1 Butterfly Conservation (Head of Monitoring), Manor Yard, East Lulworth, Wareham, Dorset BH20 5QP, UK to Arthropod conservation is clearly demonstrated in most systems and it is very encouraging to see how much research work is being done in this area.

The strong conservation message is the need to balance the benefits of increased productivity of farmed land through technical advances such as the use of pesticides, herbicides and homogenisation of habitat with the need to maintain Arthropod diversity for their intrinsic worth and for the ecosystem services they provide, such as regulating natural enemies and pollination. Habitat fragmentation is also highlighted as a major issue for Arthropod conservation and landscape-scale conservation efforts are required. Most authors advocate the need to maintain invertebrate diversity and implement integrated pest management so that crop production is sustainable and environmentally sensitive. Once again, this is a highly encouraging message, albeit challenging both to quantify and implement.

The final two book chapters focus on forest Arthropod communities and pesticide residues in crops. These seem a little out of place.

At 422 pages, there is clearly a huge amount of valuable and interesting information in this book, which is an important reference work. I thoroughly enjoyed reading it. Perhaps not surprisingly, there is some repetition in content, for example the value of Arthropods in regulating crop pest species, whilst there are inconsistencies in terms of facts such as how many insect species there are globally. More thorough editing may have helped with the latter issue.

The book is priced at $£ 119$, which reflects the volume of material presented and the fact that it is an academic book. However, the print quality is not much better than that which could be produced with a home inkjet printer, though the book is well bound and should stand the test of time. On the positive side, there are a number of colour plates of Arthropods and their habitats. 